\title{
Reconfigurable Low-Phase Noise Frequency Generation up to 92.5 GHz in a Monolithically Integrated Silicon Photonics Circuit
}

\author{
A. Malacarne(1), F. Falconi(2), A. Bigongiari(3), A. D’Errico(3), A. Bogoni(2) and C. Porzi(2) \\ (1) CNIT - National Inter-University Consortium for Telecommunications, Photonic Networks \& \\ Technologies National Laboratory, Pisa, Italy antonio.malacarne@cnit.it \\ (2) Sant'Anna School of Advanced Studies, TeCIP Institute, Pisa, Italy \\ (3) Ericsson Research, Pisa, Italy
}

\begin{abstract}
A$ silicon photonics chip is successfully employed for frequency multiplication of an $18.5-\mathrm{GHz}$ clock up to $92.5 \mathrm{GHz}(\times 5)$ with low additional phase noise. The chip includes a 20-GHz phase modulator for comb generation and a thermally tunable distributed feedback resonator for reconfigurable spectral mode selection.
\end{abstract}

\section{Introduction}

Fifth generation (5G) wireless technology in mobile communication networks plans the utilization of the $24-100 \mathrm{GHz} \mathrm{mm}$-wave range, whereas automotive radar standards already reach the $77-81 \mathrm{GHz}$ one [1]. In both examples, higher carrier frequency enables larger bandwidth (for radar this translates into better resolution, for telecom into larger data rate) and smaller antenna size. In particular, to meet the ever increasing need for larger bandwidth, $\mathrm{Wi}-\mathrm{Fi}$ standard 802.11ad already operates in the $60 \mathrm{GHz}$ regime, achieving data rates up to $7 \mathrm{~Gb} / \mathrm{s}$ [2], and $5 \mathrm{G}$ promises to provide data rates up to $10 \mathrm{~Gb} / \mathrm{s}$ by employing higher frequency [3]. The higher atmospheric attenuation of higher frequency makes this choice robust to inter-cell interference and suitable for distances up to a few kilometers. All those features are fully compatible with the emerging centralized radio access network (C-RAN) architectures where, by sharing a centralized base station for signal processing (generation, up/down conversion, etc.), remoting of small-size simplified antenna sites is possible through optical fiber links [4]. However, in such systems, phase noise (PN) of the generated $\mathrm{mm}$ wave carriers has a severe impact, especially for multicarrier signals with small subcarrier spacing, as envisioned in $5 \mathrm{G}$ wireless systems [5],[6].

In such a context, photonic techniques based on heterodyne detection of two laser carriers in a high-speed photodiode (PD) have long been proposed for generating high-frequency RF waves [7],[8], with benefits in terms of broad bandwidth offered by photonics and possibility of low-loss transport of the mm-wave signal through an optical fiber distribution network. Excellent PN performance have been achieved by selecting the beating optical modes out from a modelocked fiber laser [9]. Though, such solution prevents integration and exhibits a high complexity. Other solutions realize dualwavelength laser sources [10]. However, in those cases the use of InP platform is not particularly suitable for large volume production, the solution suffers from large phase noise [11] and/or fixed lasers frequency detuning. An alternative way to achieve PN performance comparable with a highquality lower-frequency reference clock, is to perform frequency multiplication. It can be done through electrical phase-locked loops (PLLs) [12],[13], but non-idealities of electrical/electronic circuits (parasitic effects, amplifier noise etc.) push the operating conditions far from theoretical predictions [14]. In the optical domain, two optical beating tones can be conveniently selected from an optical spectral comb (OSC) generated through electro-optic modulation of a laser source (LS), driven by a reference RF clock. In this case, the quality of the generated RF tone depends on the purity of the lower-frequency reference, whereas the optical PN and linewidth due to the LS gets canceled during the beating process at the PD [15]. Mode selection can be performed through several types of optical filter, e.g. arrayed-waveguide gratings (AWGs) [16], FabryPerot filters (FPFs) [17], liquid crystal-on-silicon (LCoS) matrixes [18] etc.. However, so far, a monolithically integrated chip including OSC generation, mode selection and recombination, has not been implemented.

In this work, we present a monolithic silicon photonic integrated circuit (PIC) for RF frequency multiplication up to the W-band $(75-110 \mathrm{GHz})$, incorporating OSC generation through highspeed phase modulator (PM), spectral mode selection through a $4^{\text {th }}$-order distributed feedback reflector (DFBR) bandpass filter [19], and subsequent recombination with the original laser mode at the PIC output. OSC generation is driven by an $18.5-\mathrm{GHz}$ reference signal, and five-fold frequency multiplication at $92.5 \mathrm{GHz}$ is successfully demonstrated with limited additional $\mathrm{PN}$ with respect to the reference. The proposed method is C-MOS compatible, mode selection can be reconfigured either by thermally tuning the 
DFBR or by tuning the laser frequency, and a complete PN and time jitter analysis is reported.

\section{On-chip implementation}

The experimental setup employed to test the PIC is reported in Fig. 1. The circuit has been implemented in silicon-on-insulator (SOI) platform through a standard multi project wafer service [20]. The temperature of the whole PIC is kept constant by a Peltier cell controlled by a thermistor-based feedback. A C-band tunable (T)LS with emission power of $14 \mathrm{dBm}$ is injected at the PIC input through an on-chip grating coupler (GC). A picture of the PIC is reported in Fig. 2. Light is then split in two arms through a multi-mode interference (MMI) splitter. The upper arm includes the PM employed for OSC generation and the tunable DFBR filter. The lower arm embeds a variable optical attenuator (VOA) consisting of a MZI structure with a thermal phase shifter on each arm. An RF synthesizer (Synth) generates a continuous wave with an $18.5 \mathrm{GHz}-$ frequency, acting as the lower-frequency clock and employed for driving the PM with a power of $27 \mathrm{dBm}$ after an RF amplifier. The SOI PM is based on plasma dispersion effect in a doped silicon rib waveguide embedding a lateral pnjunction and operates in carrier-depletion regime. To set the modulator working condition, a proper $\mathrm{DC}$ reverse bias voltage ( $3.2 \mathrm{~V}$ for maximizing the OSC generation efficiency) is coupled to the RF clock through a bias-tee. To achieve highfrequency RF carrier generation, one OSC mode is first selected by the following DFBR tunable filter and then coupled with the input laser mode through a MMI coupler. The two coupled optical

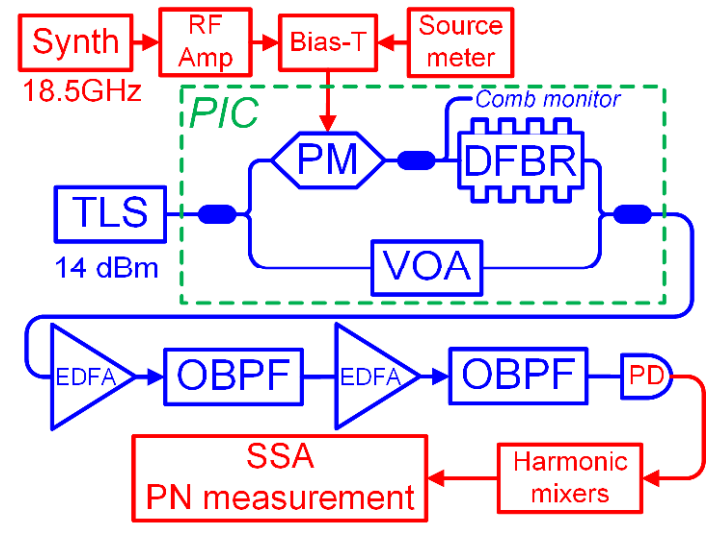

Fig. 1 Experimental setup for complete PIC testing.

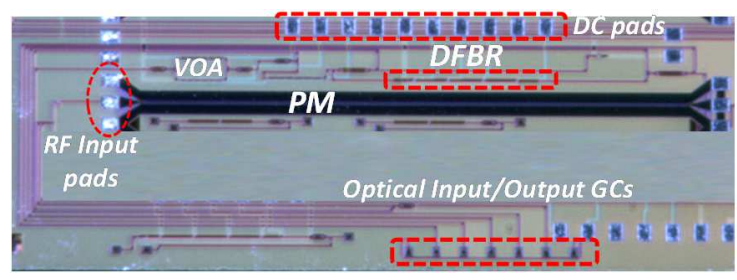

Fig. 2 Fabricated PIC.

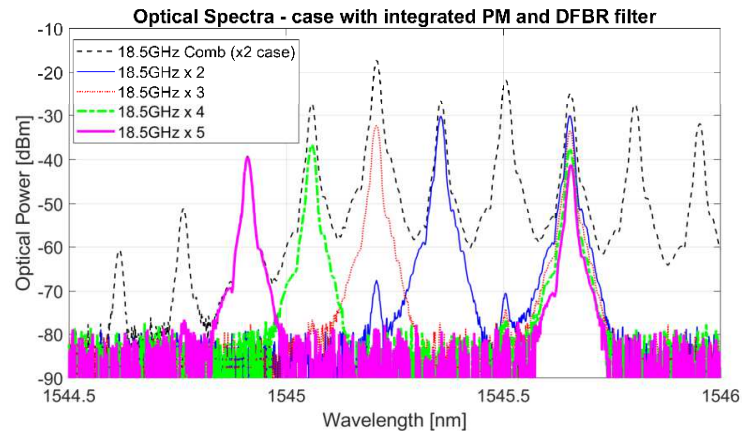

Fig. 3 OSC spectrum together with PIC output spectrum for each obtained frequency multiplication factor.

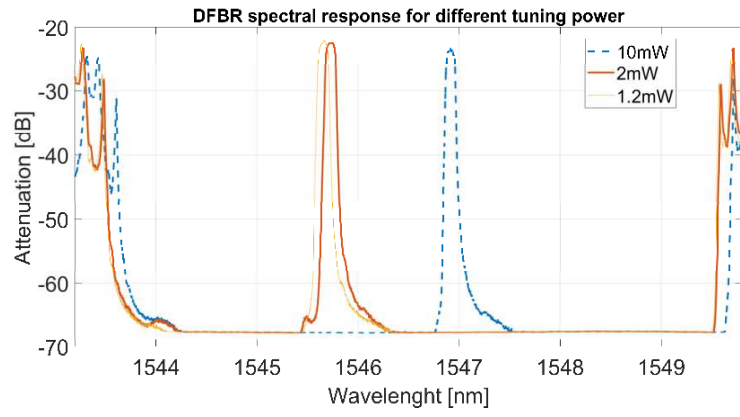

Fig. 4 DFBR filter power spectral response for different average power values dissipated on the thermal shifters.

modes are then extracted from the PIC through an output GC and delivered to a $100-\mathrm{GHz} P D$, for generating a beating RF carrier with a frequency equal to their spectral spacing, multiple of the clock frequency, i.e. at $N \cdot 18.5 \mathrm{GHz}$, being $N$ a positive integer. After photodetection, a Signal Source Analyzer (SSA) is employed (Agilent E5052A) for PN measurement. A Microwave Downconverter (E5053A) and additional external harmonic mixers are used to extend the bandwidth limitation of the instrument up to $110 \mathrm{GHz}$. To compensate for the large electrical loss due to the harmonic mixers and the high optical loss introduced by the PIC (4 dB of coupling loss at each input/output GC, $5 \mathrm{~dB}$ of PM insertion loss, $3 \mathrm{~dB}$ of loss for each traversed $\mathrm{MMI})$, a double stage of optical amplification through erbium-doped fiber amplifiers (EDFAs) and tunable optical bandpass filters (OBPF) for removing out-of-band amplified spontaneous emission, is inserted before the PD. Fig. 3 shows the optical spectrum of the generated OSC (dashed thin line) at the comb monitor port (Fig. 1). Through the DFBR filter, one of the comb mode is selected and coupled with the original laser mode. The passband filter loss is $2 \mathrm{~dB}$, with a $1 \mathrm{~dB}$-band of $10 \mathrm{GHz}$ and adjacent mode rejection higher than $35 \mathrm{~dB}$. The DFBR passband can be tuned across a range of $6 \mathrm{~nm}$, through four thermal shifters, individually heating each of the four coupled cavities in the structure [19]. An example of filter response and tunability is 
reported in Fig. 4 for three different average power values dissipated on the thermal shifters. However, for convenience, the filter spectral response was kept fixed, and the input laser was spectrally tuned with an $18.5 \mathrm{GHz}$-step, to obtain, at the PIC output, the spectra reported by Fig. 3, corresponding to a frequency multiplication factor equal to $2(37 \mathrm{GHz}), 3(55.5 \mathrm{GHz}), 4(74 \mathrm{GHz})$ and $5(92.5 \mathrm{GHz})$ (solid thin, dotted, dash-dot, solid thick line, respectively), with respect to the clock frequency. The OSC spectrum shown in Fig. 3 is the one in case of twofold frequency multiplication. Fig. 5 shows the PN power spectral density (PSD) curves for multiplication factors up to 5. PN curves are measured in a range $1 \mathrm{kHz}-40 \mathrm{MHz}$ of frequency offset from the carrier. The range has been chosen according to the requirements of modern wireless communication applications and considering the $40 \mathrm{MHz}$ instrument limit. The increasingly higher noise level at larger multiplication factor is in accordance with the theoretical dependence of the $\mathrm{dBc} / \mathrm{Hz}$ PN on the square of $\mathrm{N}$. Analyzing the excess noise in $\mathrm{dBc} / \mathrm{Hz}$ among the reference curve and each multiplied-frequency component, only part of it is due to the higher analyzed frequency. The residual discrepancy, particularly pronounced in the range $3-50 \mathrm{kHz}$, where a wide peak centered at about $20 \mathrm{kHz}$ is clearly visible, is ascribed to additional PN to the optical mode selected by the DFBR filter. In fact, local temperature fluctuations at the heaters employed for filter tuning, translates into a fluctuation of the DFBR filter spectral response. Such fluctuation translates into phase fluctuation on the selected mode, therefore leading to an additional PN term on the generated RF tone after beating between filtered and laser modes, at the PD. As a matter of fact, such additional spectral "bell-shaped" noise contribution appears to be identical for each generated RF tone whereas, in the ranges where the weight of such a disturb decreases, scaling of the PN PSD value with carrier frequency can be appreciated. As a final remark, the relatively high floor at detuning values

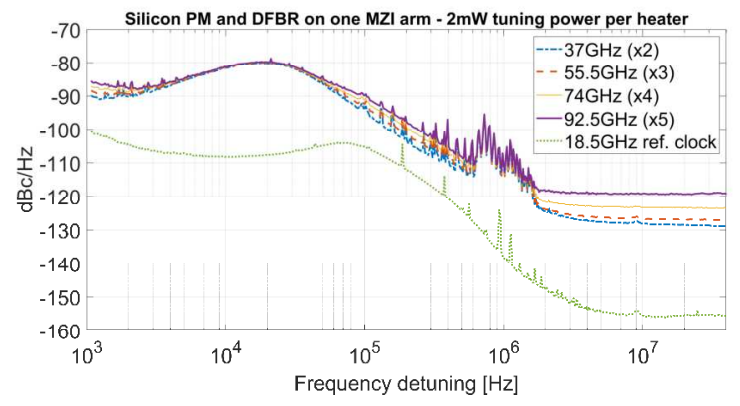

Fig. 5 Phase noise PSD of generated RF carrier at frequency of $37,55.5,74$ and $92.5 \mathrm{GHz}$, together with the one of the employed clock signal at $18.5 \mathrm{GHz}$.
$>10 \mathrm{MHz}$, represents a sensitivity limit of the measure, due to both the available signal power and its signal-to-noise ratio. The main limitation in that sense is given by the optical insertion loss of the PIC, and the consequent optical SNR decrease caused by the use of the two EDFAs.

As a last performance evaluation, root-meansquare (RMS) time jitter (TJ) for each generated RF tone was measured in the same frequency detuning range. As shown by Fig. 6, TJ value decreases as the generated frequency increases. Such a trend is expected as, for increasing carrier frequency, the weight of a constant disturb decreases. To verify that such an additional PN term is due to DFBR filter fluctuations, TJ performance has been compared with the case where the two beating modes out of the OSC (at comb monitor port, see Fig. 1) are selected by an external optical filter, i.e. a Finisar WaveShaper (WS) based on LCoS technology. As for ideal frequency multiplication, in this case $\mathrm{TJ}$ is independent of the multiplication factor. As a last check of the performance of the OSC generated into the silicon PM, the same measurement was repeated by using a commercial $\mathrm{LiNbO}_{3} \mathrm{PM}$. After mode selection by the same WS, the TJ performance included in Fig. 6 has been attested, looking identical to the silicon PM case.

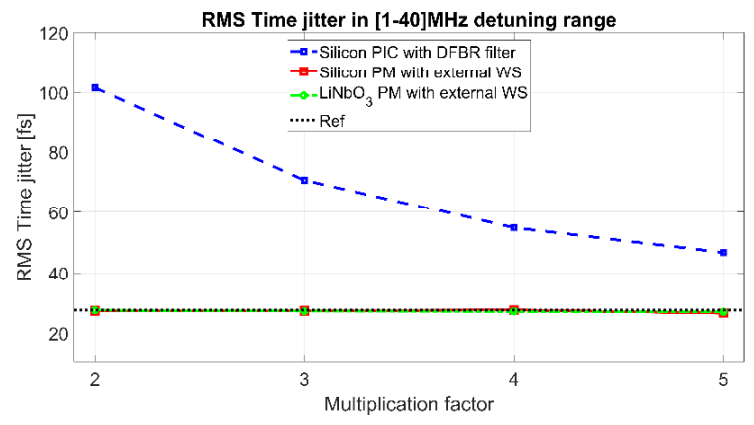

Fig. 6 RMS time jitter of traces in Fig. 5 (dashed blue square) compared with cases where an external filter selects two comb modes in case of silicon PM (solid red square) and lithium niobate PM (dash-dot green square).

\section{Conclusions}

Photonics-based frequency multiplication of an $18.5 \mathrm{GHz}$ clock up to $92.5 \mathrm{GHz}$ (fivefold) is demonstrated using a monolithic silicon chip embedding an electro-optic phase modulator and a custom distributed feedback reflector thermally tunable filter. Limited additional phase noise is observed, due to thermal fluctuations of the filter. The solution is suitable for low-loss transport of low-phase noise W-band carriers through an optical fiber distribution network.

\section{Acknowledgements}

This work has been supported by the POINTING project founded by the Italian Space Agency. 


\section{References}

[1] Final draft ETSI EN 302264 V2.1.1 (2017-02) https://www.etsi.org/deliver/etsi_en/302200_302299/3 02264/02.01.01_30/en_302264v020101v.pdf.

[2] T. Nitsche et al., "leee 802.11ad: directional $60 \mathrm{ghz}$ communication for multi-gigabit-per-second wi-fi" IEEE Commun. Mag., vol. 52, no. 12, pp. 132-141, 2014.

[3] J. G. Andrewa et al., "What will $5 \mathrm{G}$ be?" IEEE J. Sel. Areas Commun., vol. 32, no. 6, pp. 1065-1082, 2014.

[4] China Mobile Research Institute, "C-RAN: The road towards green RAN" White Paper, ver. 2.3, Sep. 2013.

[5] C. Browning et al., "Gain-switched optical frequency combs for future mobile radio-over-fiber millimeterwave systems," J. Lightw. Technol., vol. 36, no. 19, pp. 4602-4610, Oct. 2018.

[6] Ana Garcia Armada, "Understanding the effects of phase noise in orthogonal frequency division multiplexing (OFDM)," IEEE Trans. Broadcasting, vol. 47, no. 2, pp. $153-159$, June 2001

[7] A. J. Seeds, "Microwave photonics" IEEE Trans. Microw. Theory Tech., vol. 50, no. 3, pp. 877-887, 2002.

[8] T. Nagatsuma, "Generating millimeter and terahertz waves" IEEE Microw. Mag., vol. 10, no. 4, pp. 64-74, 2009.

[9] G. Serafino et al., "Phase and amplitude stability of ehf-band radar carriers generated from an active mode-locked laser" J. Lightw. Technol., vol. 29, no. 23, pp. 3551-3559, 2011.

[10] G. Carpintero et al., "Microwave photonic integrated circuits for millimeter-wave wireless communications," J. Lightw. Technol., vol. 32, no. 20, pp. 3495-3501, 2014

[11] J. Hulme et al., "Fully integrated microwave frequency synthesizer on heterogeneous silicon-iii/v," Opt. Express, vol. 25, no. 3, pp. 2422-2431, Feb 2017

[12] W. P. Robins, Phase noise in signal sources: theory and applications. vol.9. IET, 1984

[13] Collins, lan. "Phase-Locked Loop (PLL) Fundamentals", Analog Dialogue 52-07, July 2018

[14] A. Elkholy et al., "A 6.75-8.25-GHz -250-dB FoM Rapid ON/OFF Fractional-N Injection-Locked Clock Multiplier" IEEE Journal of Solid-State Circuits, vol. 53, no. 6, pp. 1818-1829, 2018.

[15] A. Hirata et al., "Low-phase noise photonic millimeterwave generator using an AWG integrated with a 3-dB combiner," 2004 IEEE International Topical Meeting on Microwave Photonics (IEEE Cat. No.04EX859), 2004, pp. 209-212.

[16] H. Song et al., "Broadband-frequency-tunable subterahertz wave generation using an optical comb, awgs, optical switches, and a uni-traveling carrier photodiode for spectroscopic applications," J. Lightw. Technol., vol. 26, no. 15, pp. 2521-2530, 2008.

[17] R. Criado et al., "Continuous-wave sub-thz photonic generation with ultra-narrow linewidth, ultra-high resolution, full frequency range coverage and high long-term frequency stability," IEEE Trans Terahertz Sci Technol., vol. 3, no. 4, pp. 461-471, 2013.

[18] Koenig et al., "Wireless sub-thz communication system with high data rate," Nature photonics, vol. 7 , no. 12, pp. 977-981, 2013.

[19] C. Porzi et al., "Silicon photonics high-order distributed feedback resonators filters," IEEE J. Quantum Electron., vol. 56, no. 1, pp. 1-9, 2020.

[20] https://www.imec-int.com/en/integrated-photonics 